Research Article

\title{
User Association and Small-Cell Base Station On/Off Strategies for Energy Efficiency of Ultradense Networks
}

\author{
Jing Gao $\mathbb{D},{ }^{1}$ Qing Ren $\mathbb{D}^{2},{ }^{2}$ Pei Shang Gu $\mathbb{D}^{2},{ }^{2}$ and Xin Song $\mathbb{D D}^{2}$ \\ ${ }^{1}$ School of Control Engineering, Northeastern University at Qinhuangdao, Hebei 066004, China \\ ${ }^{2}$ School of Computer and Communication Engineering, Northeastern University at Qinhuangdao, Hebei 066004, China \\ Correspondence should be addressed to Jing Gao; summergj@126.com
}

Received 22 March 2019; Revised 11 June 2019; Accepted 3 July 2019; Published 18 July 2019

Academic Editor: Paolo Bellavista

Copyright ( 2019 Jing Gao et al. This is an open access article distributed under the Creative Commons Attribution License, which permits unrestricted use, distribution, and reproduction in any medium, provided the original work is properly cited.

\begin{abstract}
The widespread application of wireless mobile services and requirements of ubiquitous access have resulted in drastic growth of the mobile traffic and huge energy consumption in ultradense networks (UDNs). Therefore, energy-efficient design is very important and is becoming an inevitable trend. To improve the energy efficiency (EE) of UDNs, we present a joint optimization method considering user association and small-cell base station (SBS) on/off strategies in UDNs. The problem is formulated as a nonconvex nonlinear programming problem and is then decomposed into two subproblems: user association and SBS on/off strategies. In the user association strategy, users associate with base stations (BSs) according to their movement speeds and utility function values, under the constraints of the signal-to-interference ratio (SINR) and load balancing. In particular, taking care of user mobility, users are associated if their speed exceeds a certain threshold. The macrocell base station (MBS) considers user mobility, which prevents frequent switching between users and SBSs. In the SBS on/off strategy, SBSs are turned off according to their loads and the amount of time required for mobile users to arrive at a given SBS to further improve network energy efficiency. By turning off SBSs, negative impacts on user associations can be reduced. The simulation results show that relative to conventional algorithms, the proposed scheme achieves energy efficiency performance enhancements.
\end{abstract}

\section{Introduction}

Fifth-generation mobile communication systems (5G) have been proposed recently to meet the exponentially increasing data rate demands of mobile devices such as smart phones, wearable computers, and mobile terminals $[1,2]$. Key $5 \mathrm{G}$ technologies that have been proposed to improve system performance include UDNs, massive multiple-input multiple-output (MIMO) technology, simultaneous full-duplex technology, filter bank multicarriers, and device-to-device technology $[3,4]$. In particular, UDNs are one of the most promising approaches for achieving $5 \mathrm{G}$ network market demands, improving system performance, and enhancing network coverage $[5,6]$. UDNs are an evolution of the ultradense deployment of heterogeneous networks, and they represent a new paradigm shift for future network design. This is because, at present, the deployment of MBSs is already very dense, making it very difficult to continue to increase their density.
UDNs consist of multiple small cells, including femtocells, picocells, and microcells. These small cells are deployed randomly in the MBS coverage area. Small cells are considered a promising solution for dealing with increasing data rate demands. Unlike macrocells, small cells have low power consumption and low cost and can be deployed flexibly. Furthermore, if the traffic load of a macrocell increases dramatically, small cells can offload part of this load, thereby preventing networks from overcrowding and collapsing. Moreover, small cells can cover remote blind spots with weak coverage. The deployment of ultradense small cells has been shown to improve energy efficiency [7].

However, ultradense deployment of SBSs also brings great challenges to the entire communication system. First, with an increased number of low-power SBSs, the network structure becomes increasingly complex, thus highlighting the importance of user association. Many studies have therefore focused on how to achieve the best access for users. Second, ultradense deployment of SBSs leads to extremely 
high network power consumption. One study [8] estimated that BSs account for $\sim 57 \%$ of the total power consumption of wireless networks. With the rapid growth of mobile data traffic, information and communication systems are estimated to account for $\sim 2 \%$ of global carbon dioxide emissions [9]. Therefore, green $5 \mathrm{G}$ communication systems are expected to gain importance in the future $[10,11]$. Researchers are trying to enhance networks' energy efficiency because of increasing energy prices and the need to reduce emissions [12]. Thus far, studies on small cells have primarily focused on user associations and BS on/off operations to optimize performance.

1.1. Related Work. Several user association algorithms have been proposed to improve network performance. Zhou et al. [13] proposed a joint cell activation and selection scheme for energy efficiency of UDNs and developed a three-layer iterative algorithm to solve the nonlinear mixed-integer programming problem. Boostanimehr and Bhargava [14] proposed a quality of service- (QoS-) driven distributed cell association algorithm in which users receive a number of resource blocks that is only sufficient to satisfy their QoS constraints while maximizing the sum utility of rate or minimizing the global outage probability. Ge et al. [15] proposed a joint user association and user scheduling algorithm for load balancing by exploiting a distributed convex optimization technique called the alternating direction method of multipliers. Zhou et al. [16] maximized the weighted sum of long-term rates to balance the network load and thus achieved higher load balancing gain and energy efficiency. Shen and Yu [17] investigated pricingbased BS association schemes for heterogeneous networks and proposed a distributed price update strategy based on a coordinate descent algorithm in the dual domain. Jin and Qiu [18] proposed a marginal utility-based user association algorithm that applies an almost blank subframe-based interference coordination technique. Chen et al. [19] proposed a distributed belief propagation algorithm to solve user association problems in heterogeneous networks with various user priorities. Mugume and So [20] proposed various user association schemes based on coverage probability and average rate using stochastic geometry tools.

Almost all the aforementioned works used different methods and techniques to solve the problem of user association from the perspective of users. However, there are very few efforts that consider dynamic users, but paying more attention to static users results in certain limitations. In UDN, the dense deployment of small cells provides users with a lot of association choices, while in the actual scenarios, users are mostly mobile, which will greatly affect the association between users and the BSs. Moreover, the performance of network can be further improved by performing BS on/off strategies in a certain way.

Many BS on/off strategies and joint optimization algorithms have been proposed to further optimize network performance. Yu et al. [21] proposed heuristic algorithms to reduce energy costs by switching BSs on/off dynamically based on switching energy costs and the transmission power adjustment of BSs. Kim et al. [22] proposed a joint algorithm for switching BSs on/off and user association in a heterogeneous network based on the total cost function and density of access points within the coverage of each BS; this approach reduced energy consumption greatly. Ye et al. [23] proposed a joint scheme that considers cell association and resource allocation and introduced biasing factors to achieve optimal load-aware performance. Tang et al. [24] proposed an energy-saving algorithm with joint user association, clustering, and on/off switching strategies to reduce the average network cost. Huang et al. [25] proposed a network energy efficiency scheme for UDNs by jointly considering BS on/off switching strategies and load balancing. Another study introduced user behavior predictions based on the Markov model and dual connectivitybased seamless handover procedures [26]. Park and Lim [27] proposed a power control algorithm with mobility prediction to perform remote radio head switching operations in a heterogeneous cloud radio access network (H-CRAN) to improve energy efficiency. Chai et al. [28] proposed a joint optimization method that considers both the partial spectrum reuse scheme and the BS sleep model for improving the energy efficiency of heterogeneous networks. Cai et al. [29] developed an SBS on/off switching algorithm based on optimal distance to reduce network power consumption. Abdulkafi et al. [30] investigated a small-cell sleep strategy based on traffic load in which BSs are turned off for some duration when their traffic load decreases below a certain threshold. Liu et al. [31] proposed an energy-efficient maximization problem based on coverage probability under either a random sleeping policy or a strategic sleeping policy and proposed static and dynamic user models in the strategic sleeping policy, which only considered BS on/off strategies but not the user association. Liu and Yiqun [32] proposed Mobility Model based on Velocity Classification (MM-VC) and analyzed the characteristics of phase-type distribution of cell resident time based on Markov theory. Furthermore, the sleeping strategy among small cells was applied to reduce the energy consumption of system. However, it only considered the energy consumption and ignored the throughput of the network. Moreover, it did not consider the user association.

The above works mostly paid attention to the joint optimization algorithms between BS on/off strategies and user association. However, all of the abovementioned studies did not focus sufficiently on joint optimization algorithms of user association and BS on/off strategy based on user mobility. And the user mobility can result in frequent user handovers in UDNs. It is easy to cause the situation that the BS is empty or the associated users are few in a period of time. Ignoring dynamic user behaviors when performing BS on/off strategies will result in an enormous waste of BS power consumption.

1.2. Contribution. In this study, we developed a user association strategy and a SBS on/off strategy to improve energy efficiency of UDNs. The key contributions of the paper can be summarized as follows: 
(1) In traditional system models, users are considered fixed and their mobility is ignored, resulting in severe frequent handovers when users move too rapidly in UDNs. In order to mitigate the impact of this problem, this study proposes a user association strategy in a dynamic system model that considers user mobility. We assume that there are many lowspeed mobile users but fewer motor vehicle users in the system model, which is consisted of three tiers, including MBSs, PBSs, and FBSs, and each user has an initial movement speed and direction and maintains the movement direction unchanged for a period of time. When the user speed exceeds a certain threshold, users are directly associated with the MBS. Then, other users are associated with BSs according to their utility function values under the constraints of SINR and load balancing, thus improving the network energy efficiency.

(2) SBS on/off strategy for UDNs is proposed to further improve network energy efficiency. Considering user mobility, SBSs are turned off according to their loads and the amount of time required for mobile users to arrive at the SBS while satisfying the outage probability constraints. The more time the users spend on moving to the target SBS, the earlier the SBS is turned off.

The remainder of this paper is organized as follows. Section 2 discusses the system models and problem formulation. Section 3 presents the user association algorithm and SBS on/off strategy algorithm. Section 4 presents and discusses the simulation results. Finally, Section 5 concludes this paper.

\section{Materials and Methods}

2.1. System Model. As shown in Figure 1, we consider a downlink UDN scenario with three tiers. Tier 1 consists of a traditional MBS that is deployed at the center of the UDN. Tiers 2 and 3 consist of picocell BSs (PBSs) and femtocell BSs (FBSs), respectively. " $m_{1}$ " PBSs, " $m_{2}$ " FBSs, and " $K$ " users are deployed randomly in the MBS coverage area in the network. We assume many low-speed mobile users but fewer motor vehicle users, and each user has an initial movement speed and direction.

The SINR of user $k$ from BS $m$ is as follows:

$$
\begin{aligned}
\operatorname{SINR}_{k, m} & =\frac{p_{m} L\left(d_{k, m}\right) \psi_{m}}{I_{k, j}+\delta^{2}}, \\
I_{k, j} & =\sum_{j=1, j \neq m}^{M} p_{j} L\left(d_{k, j}\right) \psi_{j},
\end{aligned}
$$

where $p_{m}$ indicates the transmission power of BS $m ; \psi_{m}$ indicates the state of BS $m$ when $\psi_{m}=1$; BS $m$ is in the "on" state; otherwise, $\psi_{m}=0 ; \delta^{2}$ is the additive white Gaussian noise (AWGN) power; $I_{k, j}$ is the interference introduced by all other BSs (except BS $m$ ); $p_{j}$ is the transmission power of

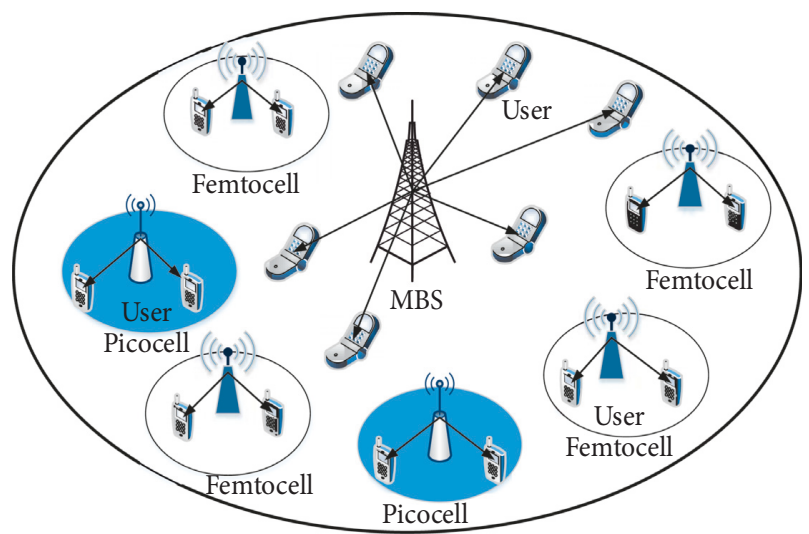

FIgURE 1: Ultradense network scenario model.

BS $j ; L(\cdot)$ is the path loss function; and $d_{k, m}$ is the distance from BS $m$ to user $k$ in meters [26].

The path loss between an MBS (or a PBS) and the user is given by

$$
L_{\mathrm{M} \& \mathrm{P}}(d)=34+40 \log _{10}(d) \mathrm{dB} .
$$

The path loss between an FBS and a user is given by

$$
L_{\mathrm{F}}(d)=37+30 \log _{10}(d) \mathrm{dB} .
$$

The rate of user $k$ can be calculated as

$$
R_{k}(\chi)=\sum_{m=1}^{M} \chi_{k, m} B \log _{2}\left(1+\operatorname{SINR}_{k, m}(\psi)\right) .
$$

When $\chi_{k, m}=1$, user $k$ is associated with BS $m$; otherwise, $\chi_{k, m}=0 . B$ represents the transmission band allocated to each user. Users can only access one BS at a time. We use the power consumption model given in [26]:

$$
E_{m}\left(\psi_{m}\right)=\varepsilon p_{m} \psi_{m}+(1-\sigma) p_{m}^{0} \psi_{m}+\sigma p_{m}^{0} .
$$

In addition to the transmission power, the total power consumption also includes the circuit power. Circuit power consumption includes the additional power consumption of devices such as mixers, filters, and digital-to-analog converters. $E_{m}\left(\psi_{m}\right)$ represents the power consumption of BS $m$; $p_{m}^{0}$ is the circuit energy consumption; $\varepsilon$ is the portion of power consumed by transmission losses; $E_{m}\left(\psi_{m}\right)=\sigma p_{m}^{0}$ is the power consumption when FBS $m$ is in the "off" state (i.e., $\left.\psi_{m}=1\right)$ and where $0 \leq \sigma<1$; and $E_{m}\left(\psi_{m}\right)=\varepsilon p_{m}+p_{m}^{0}$ is the power consumption when FBS $m$ is in the working state (i.e., $\left.\psi_{m}=0\right)$.

As in [26], we introduce an access factor $\zeta_{k}^{m}$ and define a utility function to achieve network load balancing. Here, the number of users indicates the load of BSs. The access factor can be expressed as

$$
\zeta_{k}^{m}= \begin{cases}L_{m}^{\max }-L_{m}, & L_{m}<L_{m}^{0}, \\ \frac{L_{m}^{\max }-L_{m}}{L_{m}^{\max }} \cdot \frac{L_{m}^{0}}{L_{m}}, & L_{m}<L_{m}^{0},\end{cases}
$$


where $L_{m}$ denotes the current load of BS $m, L_{m}^{0}$ is the maximum load of BS $m$ within one transmission time (equal to two slots), and $L_{m}^{\max }$ is the maximum load of BS $m$. This study uses polling scheduling. Therefore, $L_{m}^{\max }=2 L_{m}^{0}$. When a BS reaches its full load, its access factor is reduced to zero and users can no longer access this BS. The access factor controls the probability of users accessing the BS, thereby guaranteeing BS load balancing.

The utility function $U_{k}^{m}$ is expressed as

$$
U_{k}^{m}=\zeta_{k}^{m} \cdot \mathrm{EE}_{k}^{m},
$$

where $\mathrm{EE}_{k}^{m}$ represents the energy efficiency of user $k$ from BS $m$, and it is expressed as

$$
\mathrm{EE}_{k}^{m}=\frac{R_{k}^{m}}{E_{m}}=\frac{B \cdot \log _{2}\left(1+\operatorname{SINR}_{k, m}\right)}{E_{m}},
$$

where $R_{k}^{m}$ denotes the rate of user $k$ of BS $m$ and $E_{m}$ is the power consumption of BS $m$.

2.2. User Mobility Pattern. In the section, we proposed a mobile user model based on velocity classification, which is more closed to the realistic circumstance compared with general user mobility models. In the realistic circumstances, there are about four types of mobile users, namely, motor vehicle users, electric vehicle users, shared bicycle users, and pedestrian users, which have a lot of different movement characteristics. However, the most obvious is that the speed of motor vehicle users is much higher than the other three types of mobile users. Therefore, electric vehicle users, shared bicycle users, and pedestrian users are collectively referred to as low-speed mobile users.

In this paper, we assume that the realistic application scenario of the UDN is a large commercial area, where the users are almost mobile users. The mobile users tend to move more consistently in patterns owing to spatial and temporal factors. There are various rules such as traffic lights that the mobile users have to follow while moving in certain patterns. They generally move only along the designated roads, which makes their moving direction unchanged and the moving speed may change [27]. As shown in Figure 2, the mobile users are classified into two groups according to their velocity boundary $v_{\max }$, i.e., motor vehicle users $\left(v_{\text {user }}>v_{\max }\right)$ and low-speed mobile users $\left(v_{\text {user }}<v_{\max }\right)$. As in reference [32], it is assumed that the mode of users arriving to BSs obeys Poisson distribution, i.e., $X \sim P(\lambda)$, and the time interval of arriving between adjacent users obeys the exponent distribution with rate parameter of $\lambda$, i.e., $Y=\exp (\lambda)$, which are more suitable for the characteristics of such a large commercial area in urban areas. The expectation values of Poisson distribution and exponent distribution are $E(X)=\lambda$ and $E(Y)=1 / \lambda$, respectively. $E(X)$ denotes the average number of users that will reach to each BS. $E(Y)$ denotes the average time interval of arriving between adjacent users.

The movement velocity of users usually are stored in the basic information management model of the Area Control

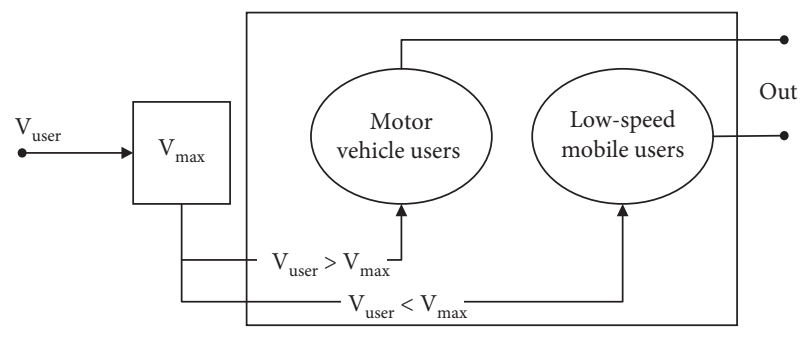

Figure 2: Mobile user model based on velocity classification.

Center (ACC) [32], which will be described in detail in the control module in the following. It is usually to determine the type of the mobile users before users associate with BSs according to the velocity. The motor vehicle users have the priority to associate with the MBS directly without considering any other conditions, which effectively avoids the frequent handovers when users move too rapidly. And different types of mobile users have different association methods, which will be described in detail in the rest of the article.

After a period of time, the closed SBSs that have applied the on/off strategy are periodically activated. Here, we only consider the network energy efficiency problem caused by the user association strategy and the SBS on/off strategy within the current cycle, ignoring the subsequent user movement and the association situation between the users and the BSs.

Figure 3 shows the position relation between mobile users and BSs [31]. User $k$ has an initial movement angle $\theta_{1, k}$ that ranges from 0 to $2 \pi$. $\theta_{2, k}$ denotes the angle between user $k$ and BS $m$. The angle between the movement angle of user $k$ and the connection between user $k$ and BS $m$ falls within the range $0<\left|\theta_{1, k}-\theta_{2, k}\right| \leq \pi / 2$, indicating that user $k$ is moving toward BS $m$. $v_{k}$ denotes the absolute velocity of user $k$. For each mobile user, the initial movement direction remains unchanged for a period of time.

2.3. Control Module. The BSs within a certain area are controlled by an Area Control Center (ACC). ACC could be deployed in Mobility Management Entity (MME) in long-term evolution system. ACC includes four models: basic information management model, state management model, traffic load monitoring model, and decision model [32]. The initial movement angles and movement speeds of users are stored in the basic information management model of ACC. During the period when the network operates, the network must clearly know the current location information and the mobile situation of the users, and all terminal users notify the network of their basic information of current locations and movement directions and record them in the MME and are periodically updated. Some basic parameters of the BSs are transmitted to the traffic load monitoring model. Therefore, the outage probability can be obtained from the traffic load monitoring model, where basic parameters make a series of calculations according to the relevant formulas. 


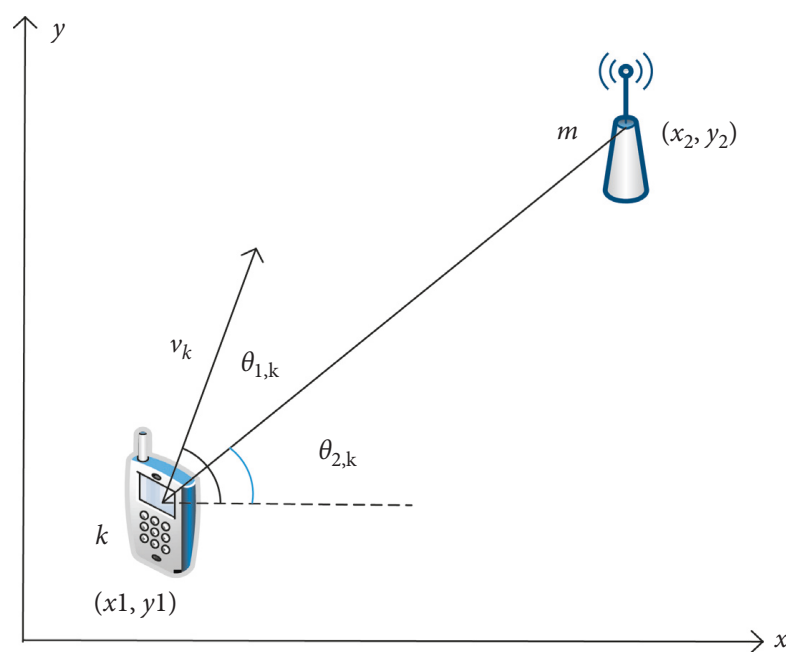

BS

8) User

FIgURE 3: Position relation between mobile user and BS.

2.4. Problem Formulation. The objective is to maximize the UDN energy efficiency while satisfying several constraints. The optimization problem is formulated as

$$
\begin{aligned}
& \max _{\psi, \chi} \quad \mathrm{EE}=\frac{\sum_{m=1}^{M} \sum_{k=1}^{K} R_{k}(\chi, \psi) \zeta_{k}^{m}}{\sum_{m=1}^{M} E_{m}(\psi)} \\
& \text { s.t. } \quad \mathrm{C} 1: \chi_{k, m} \in(0,1), \quad \forall k, m \text {, } \\
& \text { C2: } \sum_{m=1}^{M} \chi_{k, m} \leq 1, \quad \forall k \\
& \text { C3: } \psi_{m} \in(0,1), \quad \forall m \text {, } \\
& \text { C4: } \operatorname{SINR}_{k, m} \geq \operatorname{SINR}_{m}^{\text {target }}, \quad \forall k, m, \\
& \text { C5: } \sum_{k=1}^{K} \chi_{k, m} \leq L_{m}^{\max }, \quad \forall m \\
& \text { C5: } P_{\text {out }}<\xi \text {, } \\
& \text { C6: } \theta_{1, k} \in(0,2 \pi), \quad \forall k \text {. }
\end{aligned}
$$

Constraint $\mathrm{C} 1$ indicates whether user $k$ is associated with BS $m$. When $\chi_{k, m}=1$, user $k$ is associated with BS $m$; otherwise, $\chi_{k, m}=0$. Constraint $\mathrm{C} 2$ indicates that user $k$ can only access one BS at a time. Constraint $\mathrm{C} 3$ indicates the state of BS $m$; when $\psi_{m}=1$, BS $m$ is in the "on" state; otherwise, $\psi_{m}=0$. Constraint C4 ensures that if the SINR of user $k$ is larger than the threshold, user $k$ can access BS $m$. Constraint $\mathrm{C} 5$ indicates the maximum load of BS $m$, that is, $L_{m}^{\max }$. Constraint C6 indicates that the network outage probability cannot exceed a given threshold. Constraint C7 indicates that the initial movement angle of the user $k$ is $\theta_{1, k}$, which ranges from 0 to $2 \pi$.

\section{Algorithm Formulation}

This section discusses the proposed energy-efficient user association and SBS on/off strategies in UDN. Considering the nonconvex nonlinear programming problem, we decompose it into the subproblem of user association and the subproblem of SBS on/off. Then, we resolve the two subproblems individually.

3.1. User Association Strategy. Because of the ultradense deployment of SBSs in a UDN, users can make frequent handovers when moving too rapidly. However, current user association strategies do not pay sufficient attention to user mobility. Therefore, we consider user mobility in our user association strategy. When the user speed exceeds a certain threshold, the user is associated with the MBS. Additionally, other users associate with BSs according to their utility function to improve the energy efficiency of the UDN.

The specific steps are as follows:

(1) Identify users that exceed a certain speed $v_{\max }$ (i.e., motor vehicle users) and meet the minimum SINR requirements and associate them with the MBS.

(2) A set of users is established for each BS. For each user in the BS, calculate the degrees of freedom, that is, the total number of BSs that users could be associated with. Sort users by their degrees of freedom. Users with smaller degrees of freedom have higher priority when selecting a BS.

(3) Calculate the utility function value for users accessing different BSs. Users will access the BS with the largest utility function value, and the corresponding BS will update the load immediately. A user that accesses a BS will be removed from the candidate users. When a BS reaches the maximum load, other users will be prohibited from accessing that BS.

3.2. Small-Cell Base Station On/Off Strategy. To further improve the energy efficiency of UDNs, we propose using an SBS on/off strategy. Here, we only consider turning off FBSs. Because users and FBSs are randomly deployed, some FBSs may have low loads. This wastes a significant amount of energy. To solve this problem, we turn off some FBSs with low loads. Specifically, we turn off the FBSs according to the amount of time required for users to arrive at the FBSs (this is affected by user mobility). The more time the users spend moving to the target FBS, the earlier the FBS is turned off; this can also reduce user association impacts.

The SBS on/off strategy can be summarized as follows:

(1) According to the abovementioned user association strategy, the energy efficiency of the entire network and the load of each FBS should be obtained.

(2) Turn off FBSs with low load and perform a user association strategy on disconnected users. Update the energy efficiency of the UDN. Turn off FBSs until the energy efficiency is no longer increasing while 
still meeting the maximum outage probability requirements of the network. Here, the outage probability is the ratio of users not associated with any BSs to the total number of users, that is, the rate of users that do not provide the required SINR threshold SINR $k$ target to the total number of users. We denote $\xi$ as the maximum outage probability.

(3) Identify which user is moving toward each undecided FBS. Then, $\mu_{m}$ is the set of users moving toward FBS $m$. Calculate the expectation value $\lambda$ of Poisson distribution, which is the average number of users arriving to each undecided FBS. Then, calculate the total arriving time $t_{m}$ of the set of users $\mu_{m}$ of FBS $m$ according to the expectation value of exponent distribution and the number of $\mu_{m}$.

(4) Turn off FBSs based on $t_{m}$ in the declining order until the energy efficiency is no longer increasing while still meeting the maximum outage probability requirements of the network.

Traditional user association strategies are mostly based on the reference signal received power (RSRP) or SINR; that is, the user associates with the BS that can provide the maximum RSRP or the maximum SINR as the serving BS. In this study, we used the following algorithms for comparison purposes:

Maximize SINR algorithm (Max-SINR): it includes user association and SBS on/off strategies. First, users associate with BSs based on the best SINR [33]. Then, FBSs are turned off when their loads decrease below a certain threshold. In this study, the load is the number of users rather than the resource block allocated for users.

Lowest association off algorithm (LAO): it includes user association and SBS on/off strategies. First, users access BSs based on the utility function value; this value, in turn, is based on the access factor and the user's energy efficiency [25]. Then, FBSs are turned off when their loads are below a certain threshold.

Algorithm 1 shows the Max-SINR algorithm, and Algorithm 2 shows the LAO algorithm.

3.3. Complexity Analysis. In this section, the complexity of the proposed algorithm is analyzed. We assume that the number of FBSs and users is $F$ and $K$, respectively.

In Algorithm 3, lines 3-7 cost $K$ operations. We assume that $k^{\prime}$ users access the MBS. For FBS $m, k^{\prime \prime}$ candidate users are assumed. Line 9 costs $\left(K-k^{\prime}\right)$ operations. Lines $10-11$ cost $k^{\prime \prime} \cdot F$ operations. Lines $12-14$ cost $\left(1+\sum_{k=1}^{k^{\prime \prime}} \mathrm{fd}_{k}\right)$ operations. Therefore, the complexity of the proposed user association strategy algorithm is $O\left(K+\sum_{m=1}^{F}\left(K-k^{\prime}+\right.\right.$ $\left.\left.k^{\prime \prime} \cdot F+\left(1+\sum_{k=1}^{k^{\prime \prime}} \mathrm{fd}_{k}\right)\right)\right)$.

In Algorithm 4, line 3 costs 1 operation. Assuming $n$ FBSs are turned off from lines $4-7$, the cost is $n$ operations. Line 8 costs $(F-n)$ operations. Line 9 costs 1 operation. Assuming $n^{\prime}$ FBSs are turned off from lines 10-13, the cost is $n^{\prime}$ operations. Therefore, the complexity of the proposed SBSs on/off algorithm is $O\left(1+n+(F-n)+1+n^{\prime}\right)$.
In the Max-SINR algorithm, the complexity of the user association strategy algorithm is $O\left(\sum_{m=1}^{F}\left(K+k^{\prime \prime} \cdot F+(1+\right.\right.$ $\left.\left.\sum_{k=1}^{k^{\prime \prime}} \mathrm{fd}_{k}\right)\right)$ ) and that of the SBSs on/off strategy algorithm is $O(1+n)$. The LAO and Max-SINR algorithms both have the same complexity.

Therefore, the complexity of the proposed algorithm is almost the same as that of the other two algorithms. The complexity of $O(K)$ can be ignored when the number of users is within a certain range.

\section{Simulation Results}

In this section, we present the simulation results used to verify the system performance. Table 1 lists the main simulation parameters. The transmission powers of MBS, PBS, and FBS are 46, 30, and $23 \mathrm{dBm}$, respectively [34-36]. The maximum loads of MBS, PBS, and FBS within one transmission time are 50, 8, and 4, respectively. The maximum loads of MBS, PBS, and FBS are 100, 16, and 8, respectively [26]. In this simulation scenario, we assume many low-speed mobile users but fewer motor vehicle users. According to [32], the velocity boundary of low-speed mobile users and motor vehicle users is set as $30 \mathrm{~km} / \mathrm{h}$. Therefore, the speed threshold of users accessing the MBS is set as $30 \mathrm{~km} / \mathrm{h}$ $(8.3 \mathrm{~m} / \mathrm{s})$ in this study.

Figure 4 shows the users and BSs association in a downlink UDN scenario, where the pentagram represents the traditional MBS located at the center of the macrocell coverage area, asterisks represent PBSs, triangles represent FBSs, and points represent users. PBSs, FBSs, and users are deployed randomly in the MBS coverage area with $200 \mathrm{~m}$ radius. The MBS and PBSs are always "on," and the FBSs can be turned off based on the SBS on/off strategy. All BSs perform load balancing based on the maximum load capacity.

Figure 5 shows the scenario in which users associate with BSs after executing the on/off strategy. FBSs without users are turned off according to the proposed algorithm, and the users associated with these FBSs are reassociated with other BSs.

Here, we consider a downlink UDN structure with one MBS, 3 PBSs, and 18 FBSs. In the UDN, users and SBSs are randomly distributed in the MBS coverage area with $200 \mathrm{~m}$ radius.

As shown in Figure 6, our proposed algorithm had better energy efficiency than the LAO and Max-SINR algorithms even as the number of users increased. Because of user movement speeds, more users associated with the MBS. Moreover, when executing the on/off strategy, we consider the amount of time required for users to move to FBSs. This reduced UDN power consumption while effectively preventing the frequent switching of users between SBSs. In the LAO algorithm, because users associated with BSs based on energy efficiency, some FBSs with higher energy efficiency may be turned off based on their loads and the outage probability of users may increase. Therefore, the LAO algorithm had the lowest energy efficiency. The energy efficiency performance of the proposed algorithm was only $\sim 1.9 \%$ and $4.9 \%$ higher than that of the Max-SINR and LAO algorithms, respectively, for 200 users. For 260 users, the energy efficiency performance of the proposed algorithm 


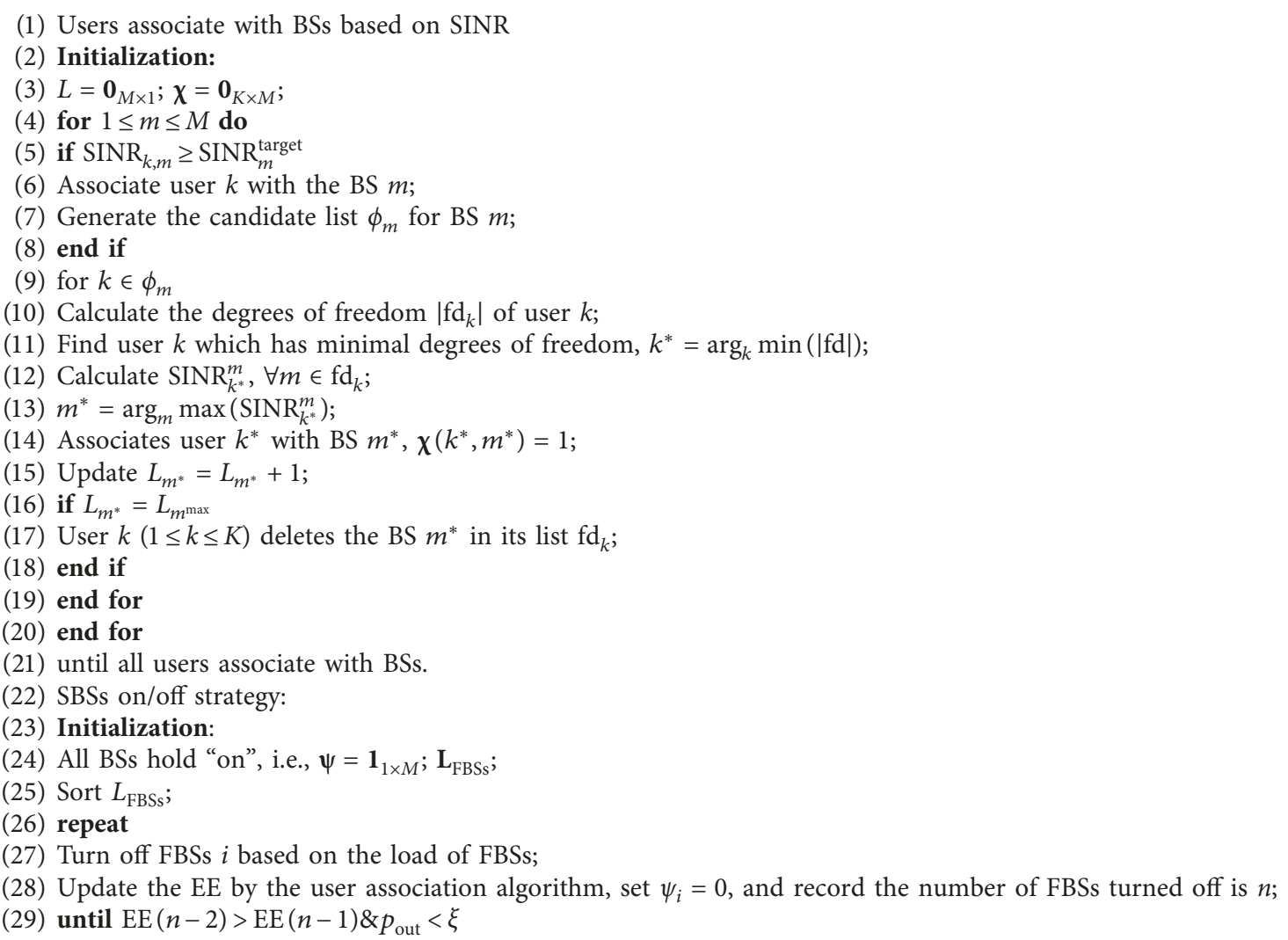

(1) Users associate with BSs based on energy efficiency

(2) Initialization:

(3) $L=\mathbf{0}_{M \times 1} ; \chi=\mathbf{0}_{K \times M}$;

(4) for $1 \leq m \leq M$ do

(5) if $\operatorname{SINR}_{k, m} \geq \operatorname{SINR}_{m}^{\text {target }}$

(6) Associate user $k$ with the BS $m$;

(7) Generate the candidate list $\phi_{m}$ for BS $m$;

(8) end if

(9) for $k \in \phi_{m}$

(10) Calculate the degrees of freedom $\left|\mathrm{fd}_{k}\right|$ of user $k$;

(11) Find user $k$ which has minimal degrees of freedom, $k^{*}=\arg _{k} \min (|\mathrm{fd}|)$;

(12) Calculate $U_{k^{*}}^{m}=\zeta_{k^{*}}^{m} \cdot E E_{k^{*}}^{m}, \forall m \in \mathrm{fd}_{k^{\prime}}$;

(13) $m^{*}=\arg _{m} \max \left(U_{k^{*}}^{m}\right)$;

(14) Associates user $k^{*}$ with BS $m^{*}, \chi\left(k^{*}, m^{*}\right)=1$;

(15) Update $L_{m^{*}}=L_{m^{*}}+1$;

(16) if $L_{m^{*}}=L_{m^{\max }}$

(17) User $k(1 \leq k \leq K)$ deletes the $\mathrm{BS} m^{*}$ in its list $\mathrm{fd}_{k}$;

(18) end if

(19) end for

(20) end for

(21) until all users associate with BSs.

(22) SBSs on/off strategy: 
(23) Initialization:

(24) All BSs hold "on", i.e., $\psi=\mathbf{1}_{1 \times M} ; \mathbf{L}_{\mathrm{FBSs}}$;

(25) Sort $L_{\mathrm{FBSs}}$;

(26) repeat

(27) Turn off FBSs $i$ based on the load of FBSs;

(28) Update the EE by the user association algorithm, set $\psi_{i}=0$, and record the number of FBSs turned off is $n$;

(29) until $\mathrm{EE}(n-2)>\mathrm{EE}(n-1) \& p_{\text {out }}<\xi$

Algorithm 2: LAO algorithm.

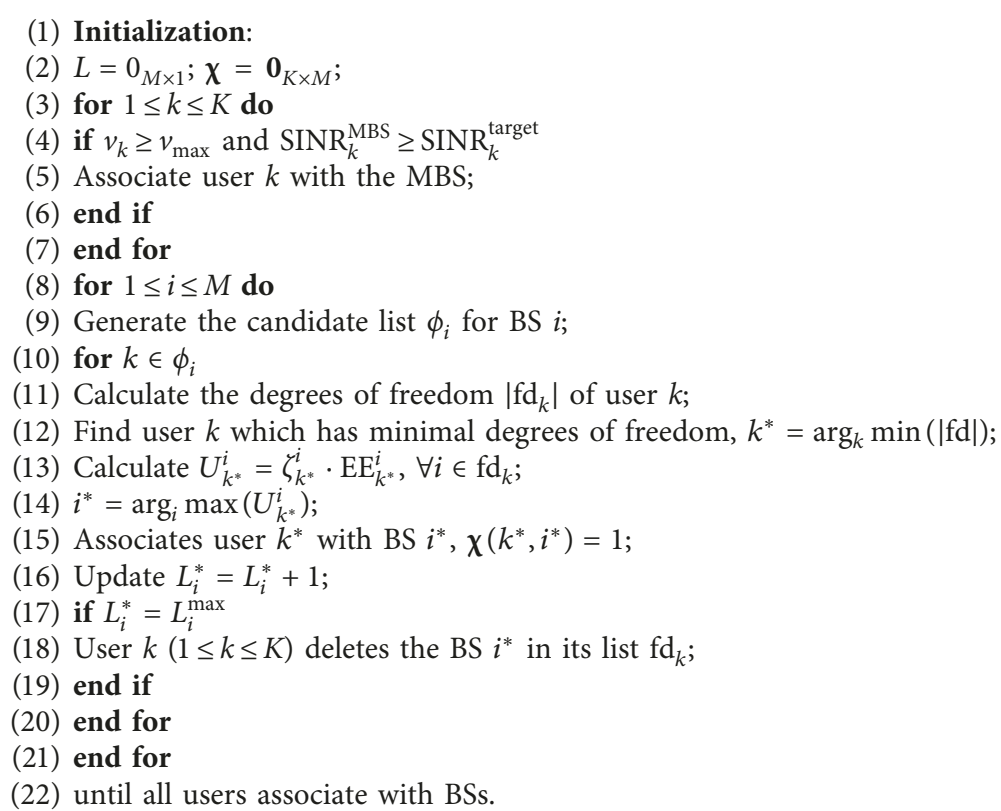

Algorithm 3: User association strategy algorithm.

(1) Initialization:

(2) All BSs hold "on", i.e., $\psi=\mathbf{1}_{1 \times M} ; \mathbf{L}_{\mathrm{FBSs}}$;

(3) Sort $L_{\mathrm{FBSs}}$;

(4) repeat

(5) Turn off FBSs $i$ base on the load of FBSs;

(6) Update the EE by the user association algorithm, set $\psi_{i}=0$, and record the number of FBSs turned off is $n$;

(7) until $\mathrm{EE}(n-2)>\mathrm{EE}(n-1) \& p_{\text {out }}<\xi$

(8) Calculate the time $t_{m}$ of each FBS;

(9) Sort $t_{m}$ in declining order;

(10) repeat

(11) Turn off FBSs $m$ base on $t_{m}$;

(12) Update the EE by user association algorithm, set $\psi_{m}=0$;

(13) until $\mathrm{EE}(n-2)>\operatorname{EE}(n-1) \& p_{\text {out }}<\xi$

Algorithm 4: SBSs on/off strategy algorithm.

was $\sim 2.1 \%$ and $4.7 \%$ higher than that of the Max-SINR and LAO algorithms, respectively.

As shown in Figure 7, the proposed algorithm produced higher network throughput than the other two algorithms even as the number of users increased. This is because some users directly associated with the MBS depending on their moving speed, and the transmission power of the MBS was significantly higher than that of the small cells. In the LAO 
TABLE 1: Simulation parameters.

\begin{tabular}{lc}
\hline Parameter & Value \\
\hline Macrocell radius & $200 \mathrm{~m}$ \\
MBS transmission power & $46 \mathrm{dBm}$ \\
PBS transmission power & $30 \mathrm{dBm}$ \\
FBS transmission power & $23 \mathrm{dBm}$ \\
MBS load $\left(L_{\mathrm{MBS}}^{0}, L_{\mathrm{MBS}}^{\max }\right)$ & $(50,100)$ \\
PBS load $\left(L_{\mathrm{PBS}}^{0}, L_{\mathrm{PBS}}^{\max }\right)$ & $(8,16)$ \\
FBS load $\left(L_{\mathrm{FBS}}^{0}, L_{\mathrm{FBS}}^{\max }\right)$ & $(4,8)$ \\
Noise power of AWGN & $-174 \mathrm{dBm} / \mathrm{Hz}$ \\
User speed threshold & $30 \mathrm{~km} / \mathrm{h}$ \\
Maximum outage probability & 0.14
\end{tabular}

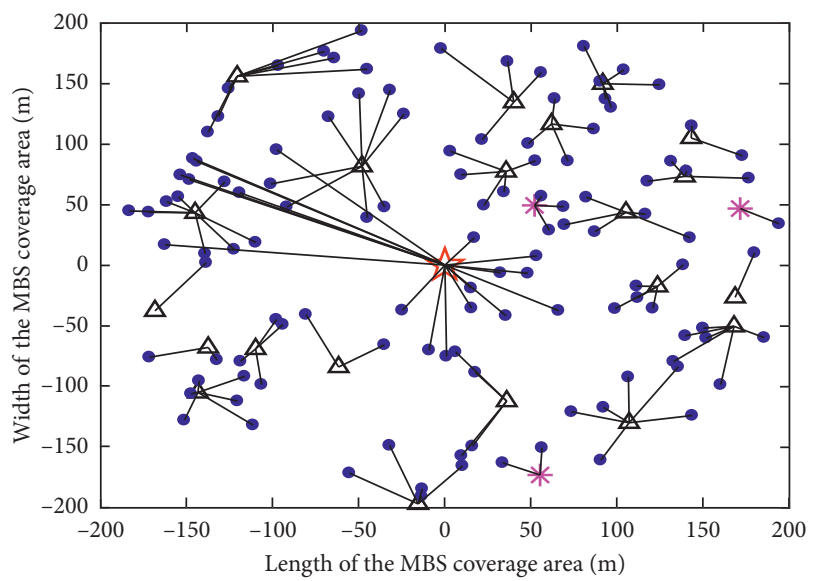

FIGURE 4: Users and BSs association.

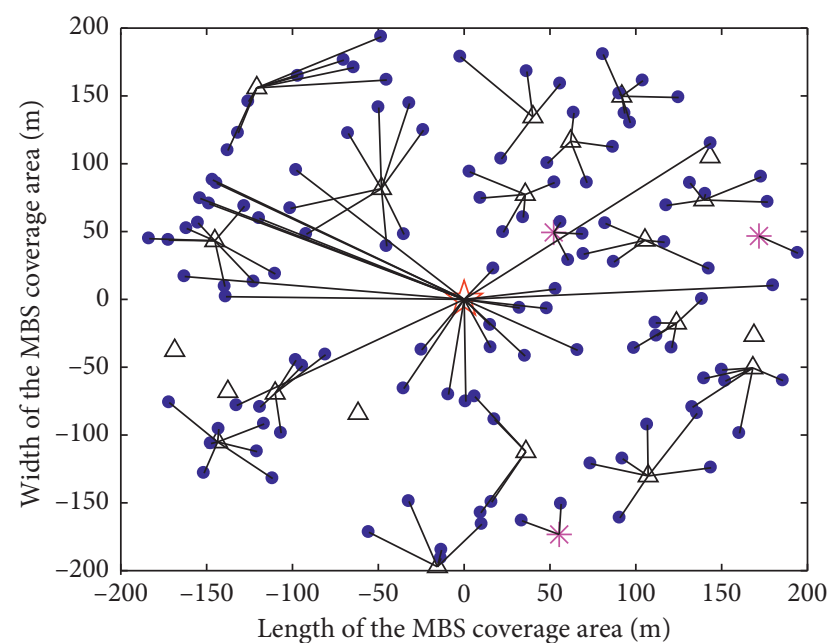

FIGURE 5: Users and BSs association after executing the on/off strategy.

algorithm, because some FBSs with higher energy efficiency may be turned off, the outage probability of users may increase when the on/off strategy is executed, resulting in the lowest network throughput among the three algorithms. The network throughput of the proposed algorithm was $\sim 2 \%$ and $4.7 \%$ higher than that of the Max-SINR and LAO algorithms, respectively, for 200 users.

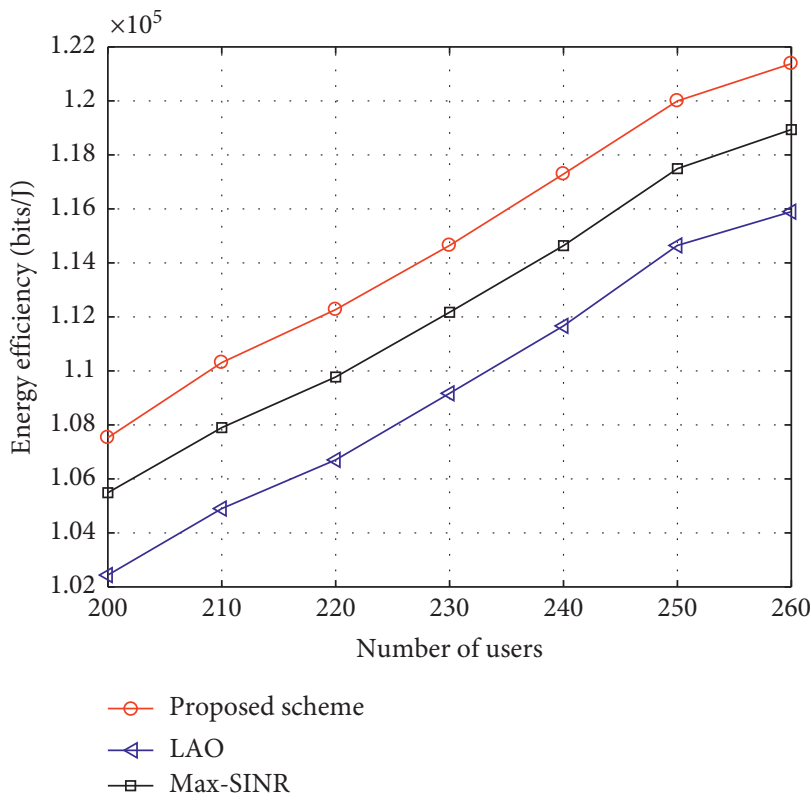

FIgURE 6: Comparison of EEs for different algorithms.

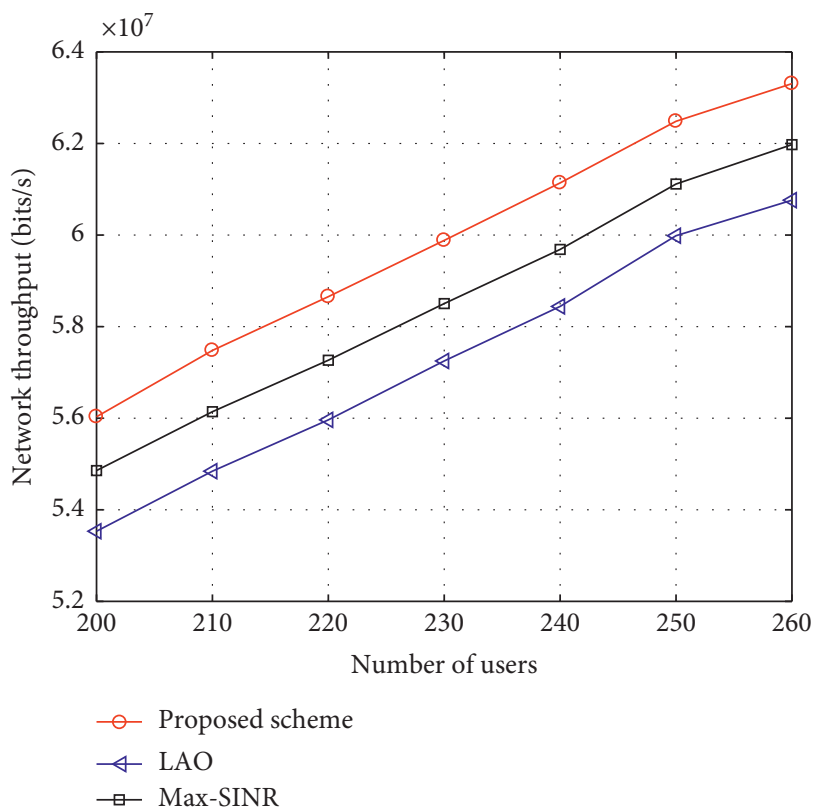

Figure 7: Comparison of throughputs for different algorithms.

As shown in Figure 8, the proposed algorithm had energy consumption similar to that of the LAO algorithm, and both of these algorithms had higher energy consumption than the Max-SINR algorithm. In the LAO algorithm, for the user association strategy, users accessed BSs based on energy efficiency. For the SBS on/off strategy, some FBSs with good performance may be turned off according to their loads, thereby reducing the network energy efficiency and increasing the outage probability of users. Once the energy efficiency of the network no longer increased and the outage probability exceeded the threshold, FBSs were no longer turned off. Therefore, fewer FBSs were turned off and 


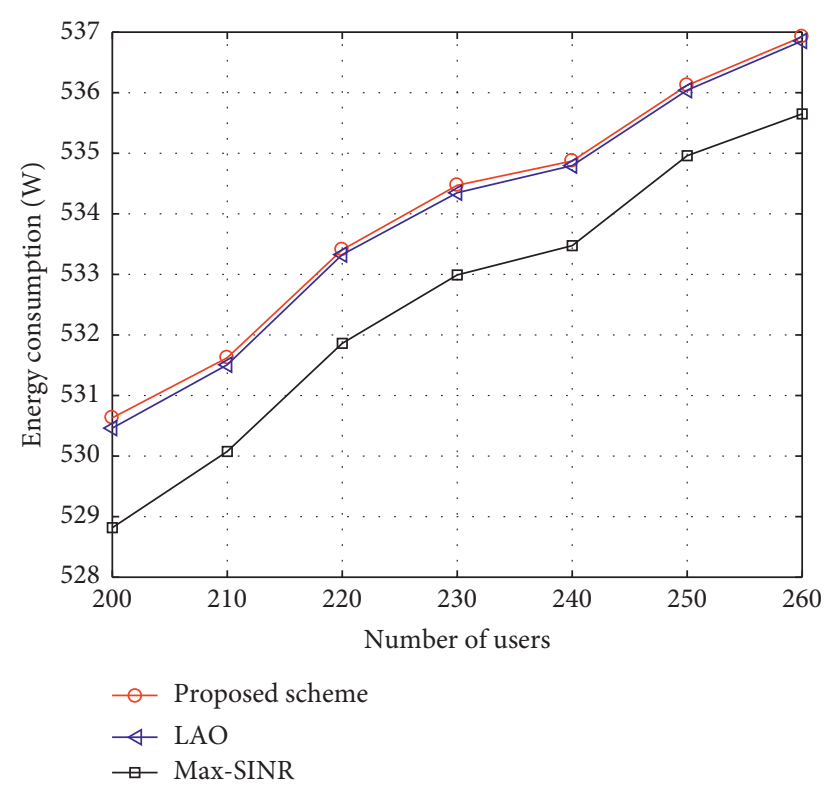

FIGURE 8: Energy consumption comparison of different algorithms.

higher energy was consumed in the LAO algorithm. The proposed algorithm had energy consumption similar to that of the LAO algorithm because user movement locations and directions were random. Turning off the corresponding FBSs may reduce the network performance. Therefore, the proposed algorithm and the LAO algorithm both had higher energy consumption than the Max-SINR algorithm.

The value of the outage probability threshold can be set from 0.05 to 0.3 generally according to different stages. The outage probability threshold is set as 0.14 in the proposed algorithm, which cannot satisfy the outage probability threshold in all stages. This paper considers the scenario that the users and SBSs are deployed densely, where users have not strictly needed low outage probability.

As shown in Figure 9, the outage probability of all algorithms fell within the required range. The proposed algorithm had lower outage probability than the other two algorithms because more users associated with the MBS and executing the on/off strategy had lesser impact on users. In the LAO algorithm, users associated with BSs based on energy efficiency and some FBSs with higher energy efficiency were turned off based on their loads, thereby affecting the reassociation of users with these FBSs. It can be observed that the outage probability increased as the number of users increased from 250 to 310 . The outage probability of the proposed algorithm was $\sim 7.7 \%$ and $1 \%$ lower than that of the LAO and Max-SINR algorithms, respectively, for 250 users. For 310 users, the outage probability of the proposed algorithm was $\sim 11 \%$ and $5.5 \%$ lower than that of the LAO and Max-SINR algorithms, respectively.

Figure 10 shows the relationship between the energy efficiency and the number of users under different MBS transmission powers. The energy efficiency increased with the number of users and with the MBS transmission power because as more users accessed the MBS with increasing MBS transmission power, more FBSs were turned off.

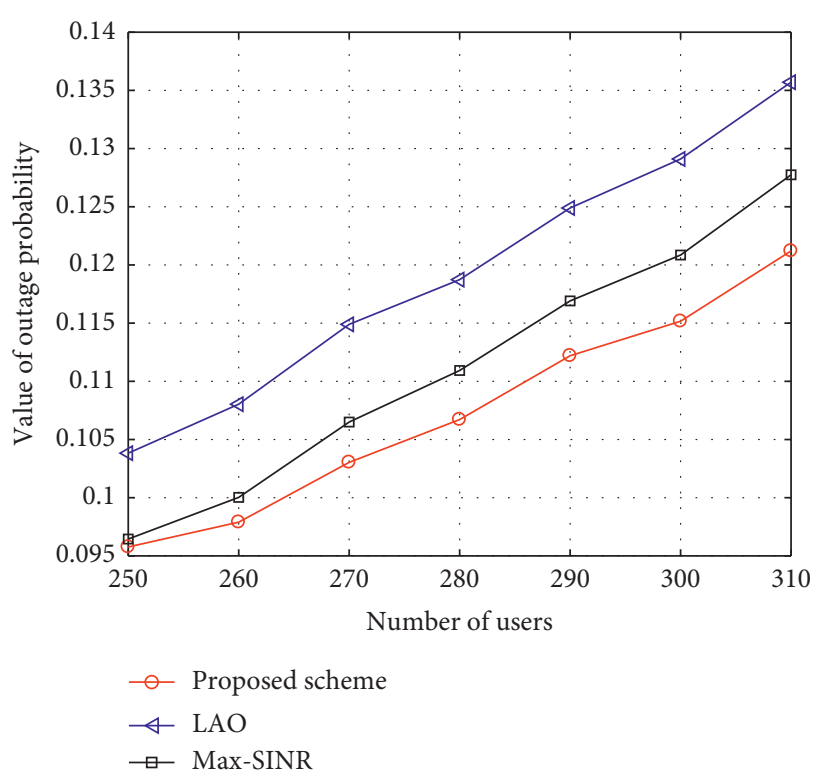

FIGURE 9: Outage probability comparison of different algorithms.

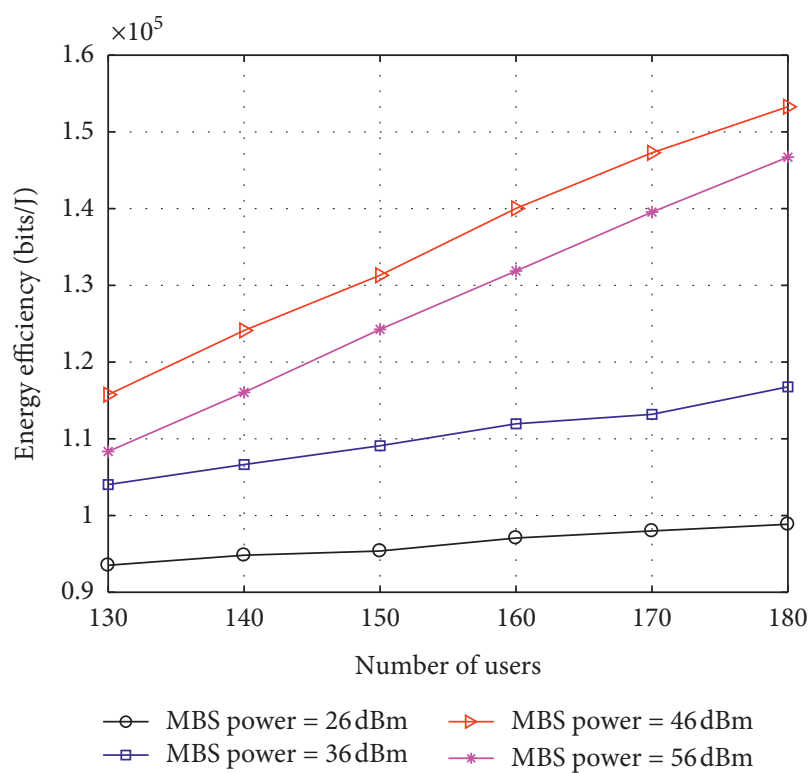

FIgure 10: Comparison of EEs for different powers of MBS.

However, when the MBS transmission power reached a certain value, the number of users associated with the MBS became stable. The energy consumption increased, and the energy efficiency accordingly decreased.

Figure 11 shows that our proposed algorithm had better energy efficiency than the LAO and Max-SINR algorithms. The reason for this is the same as that shown in Figure 5. The energy efficiency decreased as the number of FBSs increased from 14 to 24 because more network energy was consumed with increased FBS deployment and the growth rate of total network energy consumption was higher than that of total network throughput.

Figure 12 shows that the proposed algorithm produced higher network throughput than the other two algorithms. 


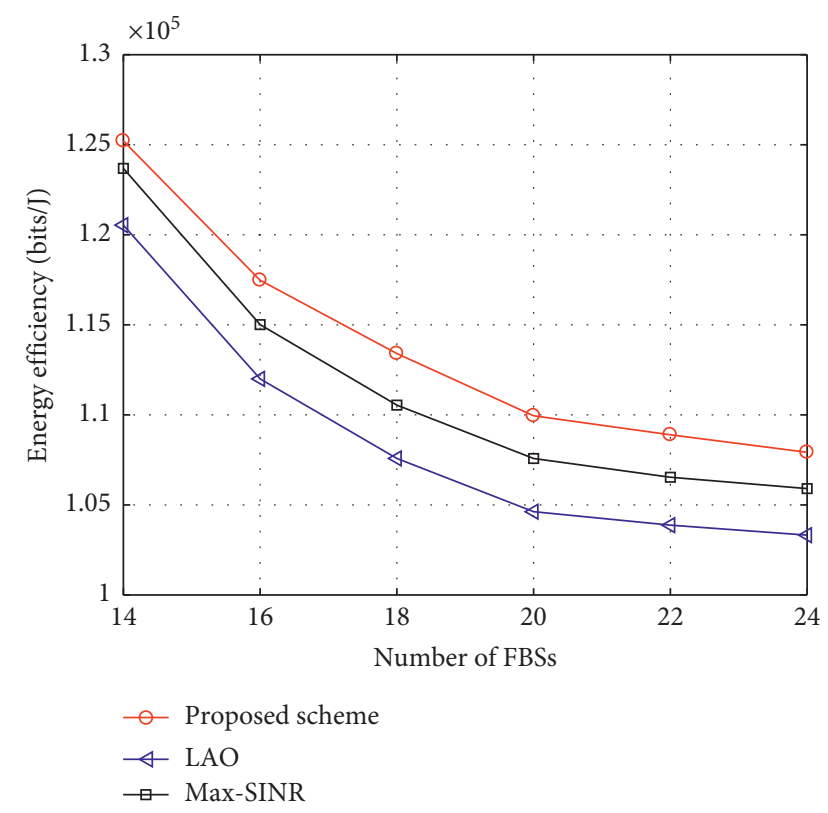

FIgURE 11: EE versus number of FBSs for different algorithms.

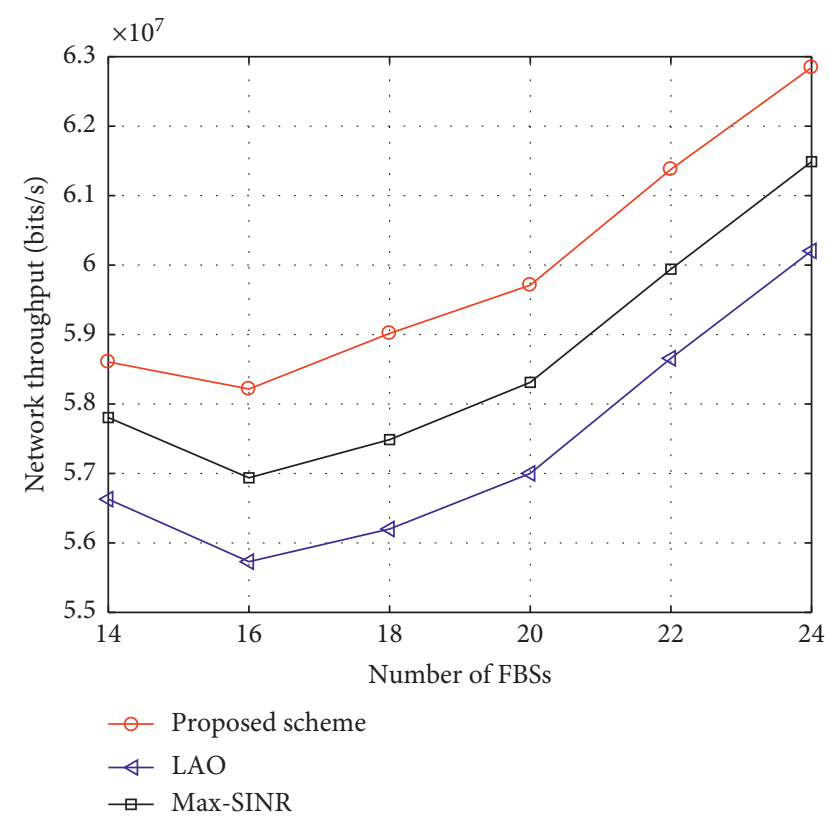

Figure 12: Throughput versus number of FBSs for different algorithms.

The reason for this is the same as that shown in Figure 6. Network throughput increased as the number of FBSs increased from 16 to 24 because more users can associate with the network and the growth rate of total network throughput was higher than that of total network energy consumption.

\section{Conclusions}

In this study, we investigated an energy-efficient user association and SBS on/off strategy for UDNs. We decomposed this nonconvex nonlinear programming problem into two subproblems. For user association, we jointly considered the SINR, load balancing, and user mobility. In our algorithm, users associated with BSs according to their movement speeds and utility function values. This optimized energy efficiency while simultaneously preventing frequent switching between users and SBSs. In the SBSs on/off scheme, FBSs were turned off according to their loads and the time at which mobile users arrived at the target FBS. This further improved the energy efficiency of UDNs while simultaneously reducing their impact on user association. The simulation results showed that the proposed strategy can optimize the network performance.

UDNs are considered a promising approach for satisfying high data rate demands. Energy efficiency has become an important system operating parameter that must be considered from the viewpoint of the economic value. However, users' quality of experience (QoE) is attracting increasing attention; the communication system performance was evaluated in terms of users' subjective perception [37]. In a future study, we will investigate how to guarantee the QoE of end users while maintaining desirable system performance in UDNs.

\section{Data Availability}

No data were used to support this study.

\section{Conflicts of Interest}

The authors declare that there are no conflicts of interest regarding the publication of this paper.

\section{Acknowledgments}

This work was supported by the National Natural Science Foundation of China (Grant nos. 61403069 and 61473066), Fundamental Research Funds for the Central Universities (no. N162304003), and the Excellent Going Abroad Experts' Training Program in Hebei Province.

\section{References}

[1] D. Soldani and A. Manzalini, "Horizon 2020 and beyond: on the $5 \mathrm{G}$ operating system for a true digital society," IEEE Vehicular Technology Magazine, vol. 10, no. 1, pp. 32-42, 2015.

[2] H. Zhang, H. Liu, J. Cheng, and V. C. M. Leung, "Downlink energy efficiency of power allocation and wireless backhaul bandwidth allocation in heterogeneous small cell networks," IEEE Transactions on Communications, vol. 66, no. 4, pp. 1705-1716, 2018.

[3] A. Gupta and R. K. Jha, "A survey of 5G network: a survey of 5G network: architecture and emerging technologies," IEEE Access, vol. 3, pp. 1206-1232, 2015.

[4] C.-X. Wang, F. Haider, X. Gao et al., "Cellular architecture and key technologies for $5 \mathrm{G}$ wireless communication networks," IEEE Communications Magazine, vol. 52, no. 2, pp. 122-130, 2014.

[5] J. Xiao, C. Yang, A. Anpalagan, Q. Ni, and M. Guizani, "Joint interference management in ultra-dense small-cell networks: a multi-domain coordination perspective," IEEE Transactions on Communications, vol. 66, no. 11, pp. 5470-5481, 2018. 
[6] T. Bilen, B. Canberk, and K. R. Chowdhury, "Handover management in software-defined ultra-dense 5G networks," IEEE Network, vol. 31, no. 4, pp. 49-55, 2017.

[7] H. Wang, S. Chen, M. Ai, and H. Xu, "Localized mobility management for $5 \mathrm{G}$ ultra dense network," IEEE Transactions on Vehicular Technology, vol. 66, no. 9, pp. 8535-8552, 2017.

[8] Q. Liu and J. Shi, "Base station sleep and spectrum allocation in heterogeneous ultra-dense networks," Wireless Personal Communications, vol. 98, no. 4, pp. 3611-3627, 2018.

[9] C. C. Coskun and E. Ayanoglu, "Energy- and spectral-efficient resource allocation algorithm for heterogeneous networks," IEEE Transactions on Vehicular Technology, vol. 67, no. 1, pp. 590-603, 2018.

[10] M. M. Mowla, I. Ahmad, D. Habibi, and Q. V. Phung, "A green communication model for 5G systems," IEEE Transactions on Green Communications and Networking, vol. 1, no. 3, pp. 264-280, 2017.

[11] K. Davaslioglu and E. Ayanoglu, "Quantifying potential energy efficiency gain in green cellular wireless networks," IEEE Communications Surveys \& Tutorials, vol. 16, no. 4, pp. 2065-2091, 2014.

[12] D. Feng, C. Jiang, G. Lim, L. J. Cimini, G. Feng, and G. Y. Li, "A survey of energy-efficient wireless communications," IEEE Communications Surveys \& Tutorials, vol. 15, no. 1, pp. 167-178, 2013.

[13] T. Zhou, N. Jiang, Z. Liu, and C. Li, "Joint cell activation and selection for green communications in ultra-dense heterogeneous networks," IEEE Access, vol. 6, pp. 1894-1904, 2018.

[14] H. Boostanimehr and V. K. Bhargava, "Unified and distributed QoS-driven cell association algorithms in heterogeneous networks," IEEE Transactions on Wireless Communications, vol. 14, no. 3, pp. 1650-1662, 2015.

[15] X. Ge, X. Li, H. Jin, J. Cheng, and V. C. M. Leung, "Joint user association and user scheduling for load balancing in heterogeneous networks," IEEE Transactions on Wireless Communications, vol. 17, no. 5, pp. 3211-3225, 2018.

[16] T. Zhou, Z. Liu, J. Zhao, C. Li, and L. Yang, "Joint user association and power control for load balancing in downlink heterogeneous cellular networks," IEEE Transactions on Vehicular Technology, vol. 67, no. 3, pp. 2582-2593, 2018.

[17] K. Shen and W. Yu, "Distributed pricing-based user association for downlink heterogeneous cellular networks," IEEE Journal on Selected Areas in Communications, vol. 32, no. 6, pp. 1100-1113, 2014.

[18] Y. Jin and L. Qiu, "Joint user association and interference coordination in heterogeneous cellular networks," IEEE Communications Letters, vol. 17, no. 12, pp. 2296-2299, 2013.

[19] Y. Chen, J. Li, Z. Lin, G. Mao, and B. Vucetic, "User association with unequal user priorities in heterogeneous cellular networks," IEEE Transactions on Vehicular Technology, vol. 65, no. 9, pp. 7374-7388, 2016.

[20] E. Mugume and D. K. C. So, "User association in energyaware dense heterogeneous cellular networks," IEEE Transactions on Wireless Communications, vol. 16, no. 3, pp. 1713-1726, 2017.

[21] N. Yu, Y. Miao, L. Mu, H. Du, H. Huang, and X. Jia, "Minimizing energy cost by dynamic switching on/off base stations in cellular networks," IEEE Transactions on Wireless Communications, vol. 15, no. 11, pp. 7457-7469, 2016.

[22] S. Kim, S. Choi, and B. G. Lee, "A joint algorithm for base station operation and user association in heterogeneous networks," IEEE Communications Letters, vol. 17, no. 8, pp. 1552-1555, 2013.
[23] Q. Ye, B. Rong, Y. Chen, M. Al-Shalash, C. Caramanis, and J. G. Andrews, "User association for load balancing in heterogeneous cellular networks," IEEE Transactions on Wireless Communications, vol. 12, no. 6, pp. 2706-2716, 2013.

[24] L. Tang, W. Wang, Y. Wang, and Q. Chen, "An energy-saving algorithm with joint user association, clustering, and on/off strategies in dense heterogeneous networks," IEEE Access, vol. 5, pp. 12988-13000, 2017.

[25] X. G. Huang, Z. F. Zhang, W. P. Dai et al., "Energy-efficient femtocells active/idle control and load balancing in heterogeneous networks," in Proceedings of the EAI International Conference Communications and Networking, pp. 237-247, Chongqing, China, September 2016.

[26] X. Huang, S. Tang, Q. Zheng, D. Zhang, and Q. Chen, "Dynamic femtocell gNB on/off strategies and seamless dual connectivity in 5G heterogeneous cellular networks," IEEE Access, vol. 6, pp. 21359-21368, 2018.

[27] H. Park and Y. Lim, "Energy-effective power control algorithm with mobility prediction for $5 \mathrm{G}$ heterogeneous cloud radio access network," Sensors (Basel), vol. 18, no. 9, p. 2904, 2018.

[28] X. Chai, Z. Zhang, and K. Long, "Joint spectrum-sharing and base station sleep model for improving energy efficiency of heterogeneous networks," IEEE Systems Journal, vol. 12, no. 1, pp. 560-570, 2018.

[29] S. Cai, Y. Che, L. Duan, J. Wang, S. Zhou, and R. Zhang, "Green 5G heterogeneous networks through dynamic smallcell operation," IEEE Journal on Selected Areas in Communications, vol. 34, no. 5, pp. 1103-1115, 2016.

[30] A. A. Abdulkafi, T. S. Kiong, D. Chieng, A. Ting, and J. Koh, "Energy efficiency improvements in heterogeneous network through traffic load balancing and sleep mode mechanisms," Wireless Personal Communications, vol. 75, no. 4, pp. 21512164, 2014.

[31] C. Liu, B. Natarajan, and H. X. Xia, "Small cell base station sleep strategies for energy efficiency," IEEE Transactions on Vehicular Technology, vol. 65, no. 3, pp. 1652-1661, 2016.

[32] L. Qi and L. Yiqun, "Energy conservation schemes based on mobility modeling in small cell networks," in Proceedings of the 2014 XXXIth URSI General Assembly and Scientific Symposium (URSI GASS), pp. 1-4, Piscataway, NJ, USA, August 2014.

[33] J. G. Andrews, "Seven ways that HetNets are a cellular paradigm shift," IEEE Communications Magazine, vol. 51, no. 3, pp. 136-144, 2013.

[34] W. Wang and G. Shen, "Energy efficiency of heterogeneous cellular network," in Proceedings of the IEEE 72nd Vehicular Technology Conference-Fall, pp. 1-5, Ottawa, ON, Canada, September 2010.

[35] D. Lopez-Perez, I. Guvenc, G. D. L. Roche, M. Kountouris, T. Quek, and J. Zhang, "Enhanced intercell interference coordination challenges in heterogeneous networks," IEEE Wireless Communications, vol. 18, no. 3, pp. 22-30, 2011.

[36] J. Zhao, Y. Liu, K. K. Chai, A. Nallanathan, Y. Chen, and Z. Han, "Spectrum allocation and power control for nonorthogonal multiple access in HetNets," IEEE Transactions on Wireless Communications, vol. 16, no. 9, pp. 5825-5837, 2017.

[37] D. Wu, Q. Wu, Y. Xu, and Y.-C. Liang, "QoE and energy aware resource allocation in small cell networks with power selection, load management, and channel allocation," IEEE Transactions on Vehicular Technology, vol. 66, no. 8, pp. 7461-7473, 2017. 


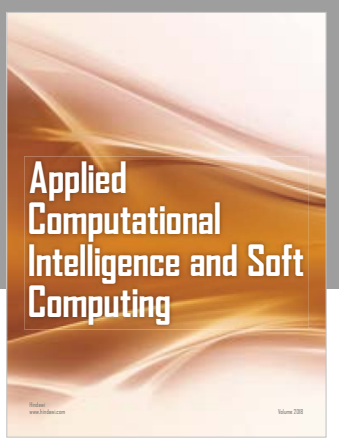

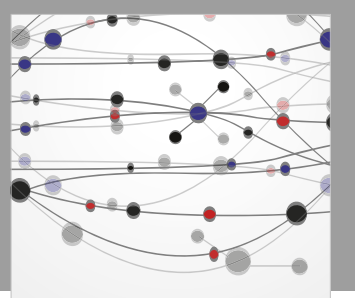

The Scientific World Journal
Submit your manuscripts at

Computing
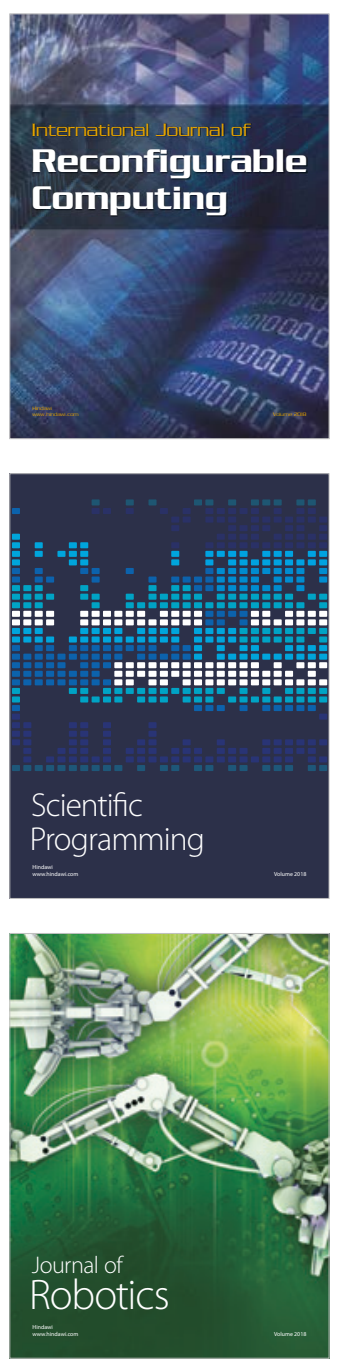

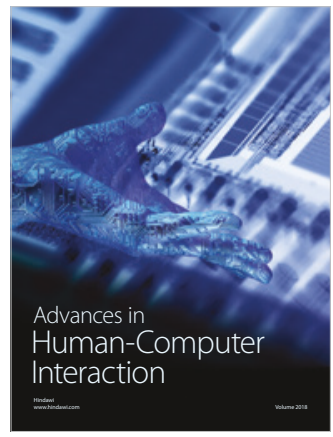

Human-Compute

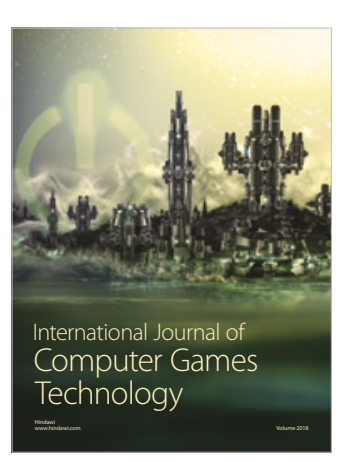

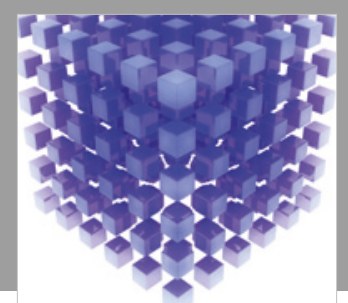

Mathematical Problems in Engineering

\section{Engincering}
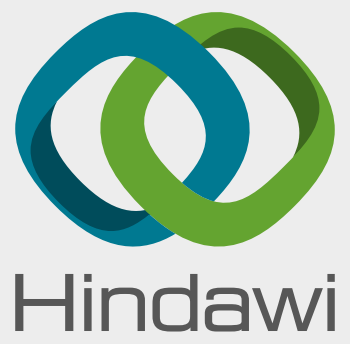

www.hindawi.com
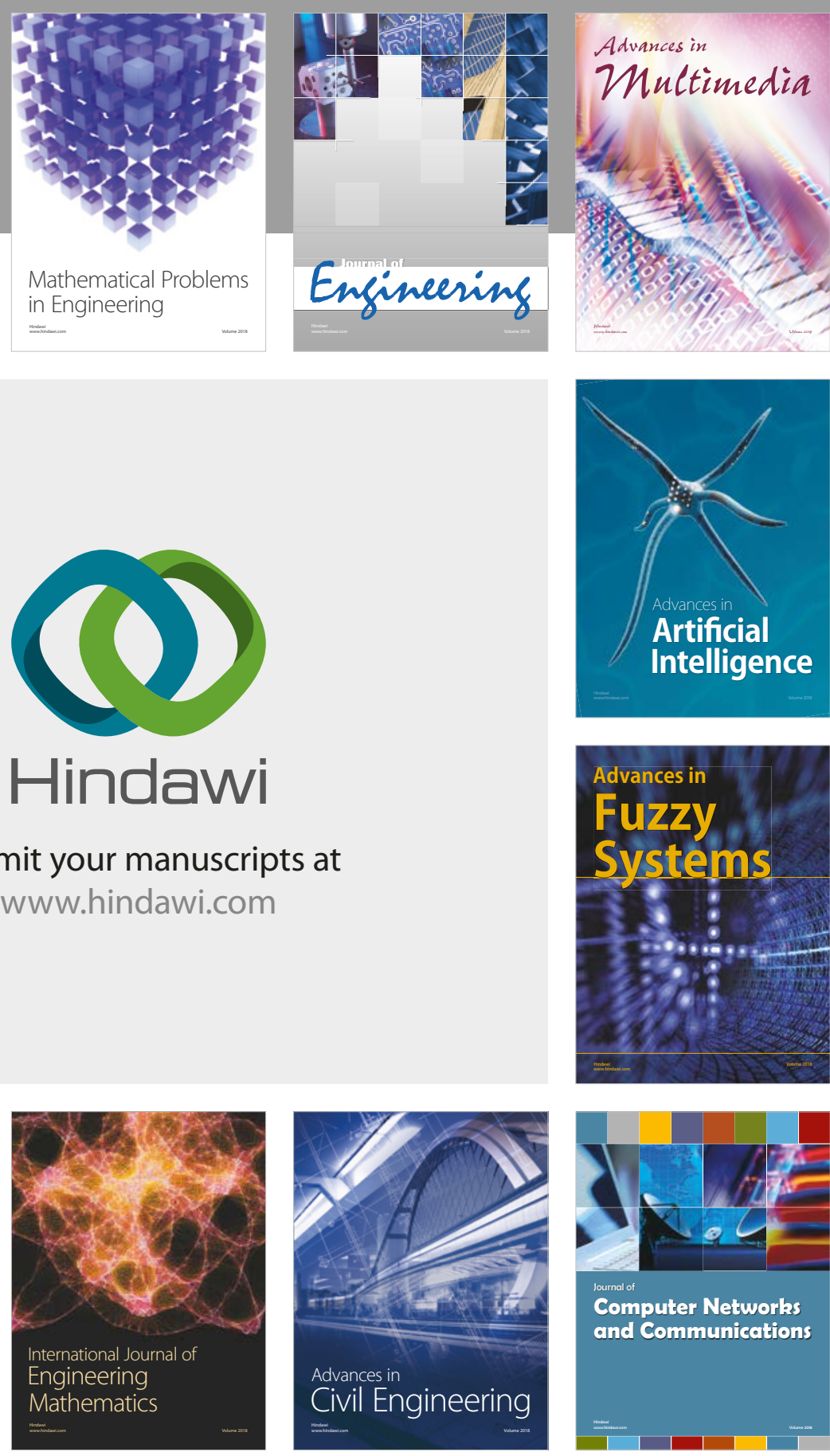

Computer Networks and Communications

Multimedia
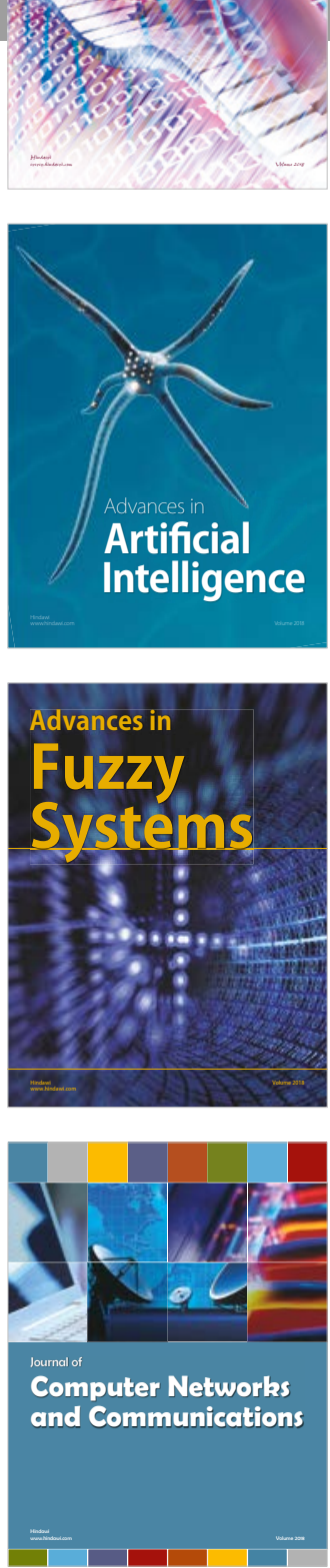

Advances in

Modelling \&

Simulation

in Engineering

interaction

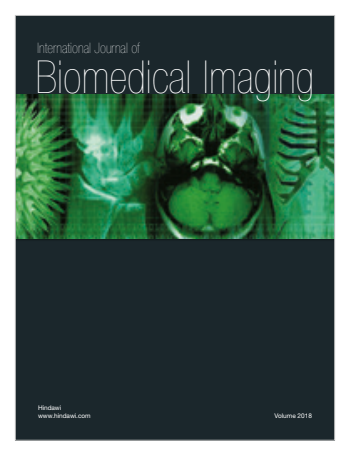

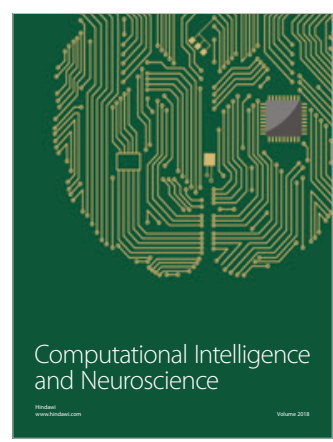

